\title{
A Rare Case of Clear Cell Adenocarcinoma of the Cervix with No Intrauterine Diethylstilbestrol Exposure
}

\author{
Vinay Mathew Thomas ${ }^{1}$, Swetha Ann Alexander ${ }^{2}$, Matthew J. Hadfield ${ }^{1}$, James Vredenburgh ${ }^{3}$ \\ 1. Internal Medicine, University of Connecticut Health Center, Farmington, USA 2. Internal Medicine, University of \\ Connecticut, Farmington, USA 3. Hematology and Oncology, Saint Francis Hospital and Medical Center, Hartford, USA
}

Corresponding author: Vinay Mathew Thomas, vmt2009@gmail.com

\begin{abstract}
Cervical cancer is the fourth most common cancer in females. Clear cell adenocarcinoma of the cervix is an uncommon histological variant and is usually seen with intrauterine exposure to diethylstilbestrol. A 28year-old female with no intrauterine exposure to diethylstilbestrol presented with postcoital bleeding. A pelvic exam revealed a cervical mass. Imaging confirmed the cervical mass and positron emission tomography scan showed an increased uptake in the cervical mass as well as the para-aortic and pelvic lymph nodes. Biopsy showed a clear cell carcinoma of the cervix. She was treated with cisplatin and paclitaxel for eight cycles and concurrent radiation therapy. She had a complete response to therapy and has been in complete remission nine months from the end of therapy. There are no clear guidelines for the treatment of clear cell carcinoma with current therapy based on the treatment of squamous and non-clear cell adenocarcinoma. Cisplatin and paclitaxel could be an option, given the successful treatment of the patient in our case.
\end{abstract}

Received 04/13/2020 Review began 04/14/2020 Review ended 04/15/2020 Published 04/23/2020

() Copyright 2020 Mathew Thomas et al. This is an open access article distributed under the terms of the Creative Commons Attribution License CC-BY 4.0., which permits unrestricted use, distribution, and reproduction in any medium, provided the original author and source are credited.
Categories: Obstetrics/Gynecology, Pathology, Oncology

Keywords: cervical clear cell carcinoma, clear cell adenocarcinoma of the cervix, clear cell cancer, cervical cancer, diethylstilbestrol, cisplatin, paclitaxel

\section{Introduction}

Cervical cancer poses a significant toll on the global cancer scene, being the fourth most common cancer in females. Cervical tumors arising from the ectocervix are most commonly squamous cell carcinomas and those arising from the endocervix are commonly adenocarcinomas. Clear cell carcinoma is a less common histological variant [1]. Clear cell adenocarcinoma of the cervix (CCAC) has classically been associated with intrauterine exposure to diethylstilbestrol (DES) [2]. However, there have been reported cases of clear cell carcinoma of the cervix without any identifiable exposure to DES. The etiology and pathogenesis associated with CCAC remain unclear. The presentation is variable, with vaginal bleeding being a common presentation [3]. Since it presents in young females, it can sometimes be misdiagnosed as functional vaginal bleeding [4]. This can often result in a delay in diagnosis. Because of the rarity of the condition, there are no established guidelines for the treatment. Current treatment methods are derived from the experience of treatment with squamous cell and non-clear cell adenocarcinomas. Depending on the stage of the disease, fertility-preserving treatment can also be tried [5].

We present a patient with CCAC who presented with postcoital bleeding and successfully completed treatment with weekly cisplatin and paclitaxel in combination with radiation therapy.

\section{Case Presentation}

A 28-year-old female with no significant past medical history presented to her gynecologist with postcoital bleeding. A pap smear was performed that revealed a normal-appearing cervix. Over the next several months, the patient began having vaginal bleeding more frequently, occurring almost daily. A pelvic exam performed at that time revealed a cervical mass, around $6 \mathrm{~cm}$. A pap smear was performed, and there was abnormal histology showing atypical glandular cells, suspicious for malignancy. HPV (human papillomavirus) testing was negative. A uterine ultrasound was ordered, which showed the uterus measuring $3.67 \times 5.54 \times 4.88 \mathrm{~cm}$, endometrium $3.41 \mathrm{~mm}$, cervix $3.04 \mathrm{~cm}$, right ovary $1.6 \times 3.66 \times 1.94 \mathrm{~cm}$, and left ovary $1.58 \times 3.16 \times 1.69 \mathrm{~cm}$. Echogenic fluid was noted in the cervical region with no free fluid identified. A biopsy of the mass showed large neoplastic cells with ovoid nuclei and clear cytoplasm, consistent with clear cell carcinoma (Figure 1). 


\section{Cureus}

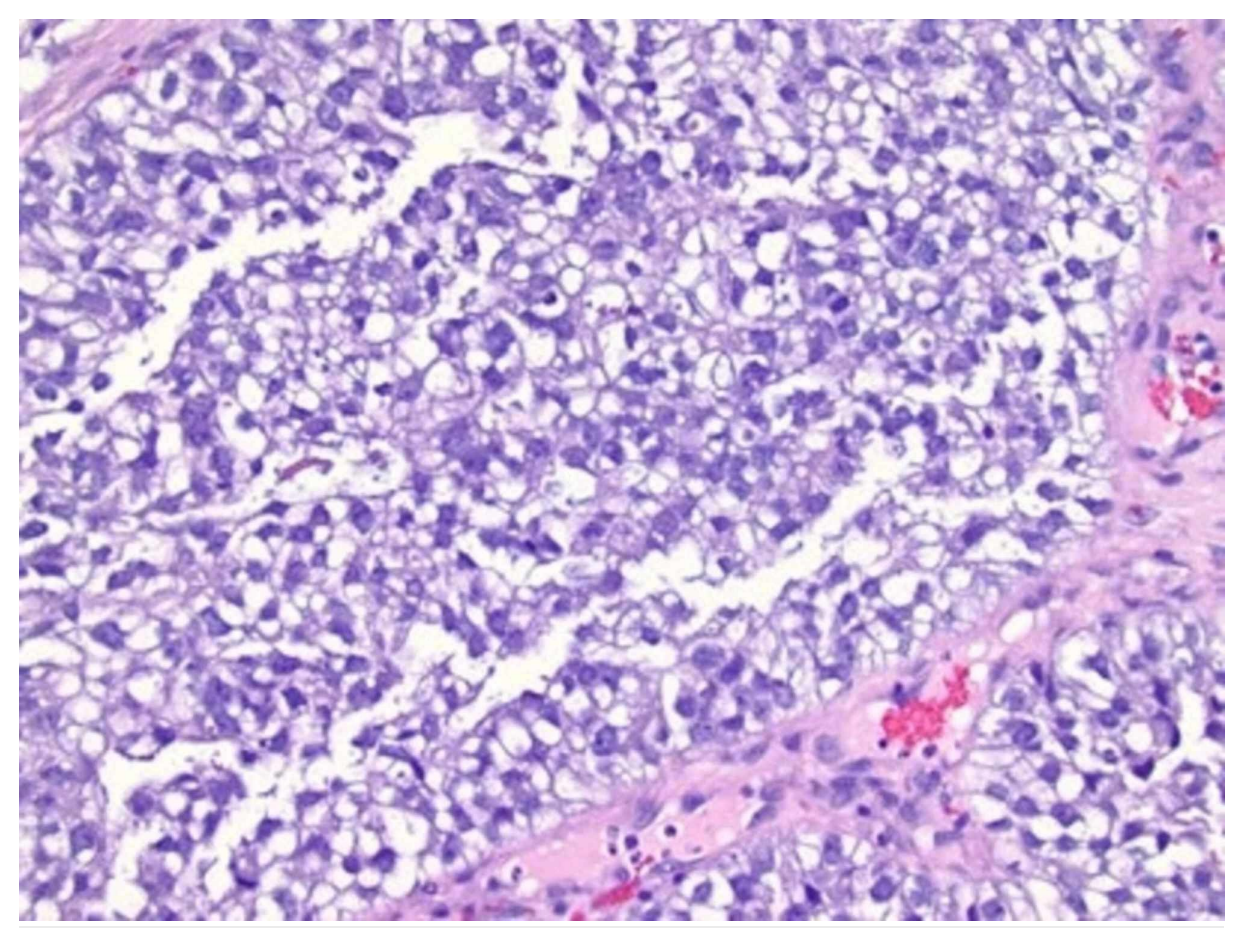

FIGURE 1: Biopsy of the cervical mass showing large neoplastic cells with ovoid nuclei and clear cytoplasm consistent with clear cell carcinoma

Immunomarkers were negative for p16, Vimentin, CD10, CDX2, CK20, Napsin A, and EFP. Periodic acidSchiff was strongly positive in the cytoplasm consistent with glycogen, which again pointed toward clear cell carcinoma. The patient's mother did not have a history of DES exposure in utero. The patient was born several years after the FDA ban on DES use in pregnancy, which made this history reliable. The patient denied risk factors such as multiple sex partners, HPV infection in the past, and smoking. Pelvic MRI was performed to further delineate the mass. The MRI showed a cervical mass measuring $6.5 \times 5.6 \times 4 \mathrm{~cm}$ projecting in the vagina with no parametrial invasion (Figure 2). 


\section{Cureus}

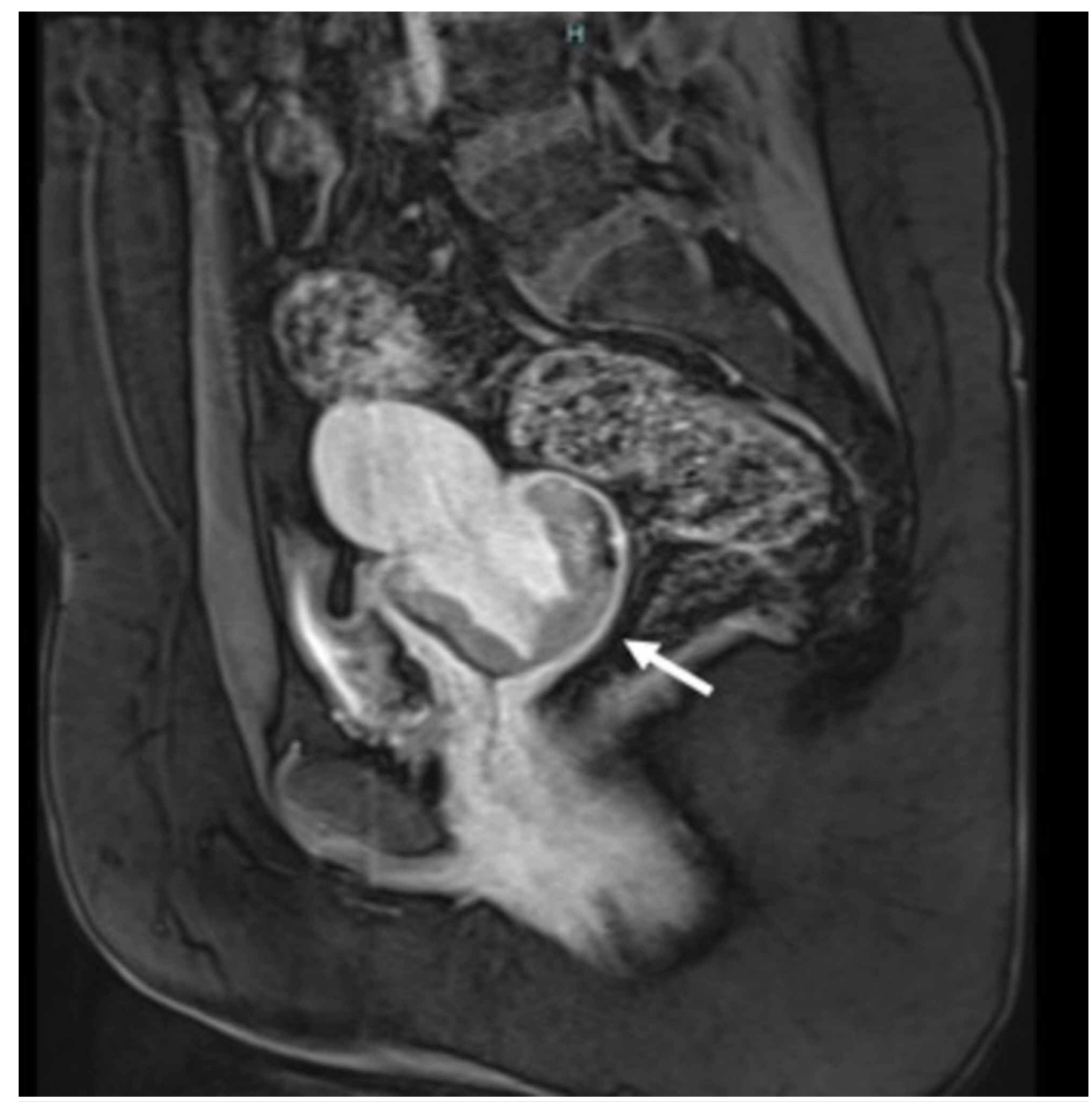

FIGURE 2: Pelvic MRI before treatment, showing the cervical mass projecting into the vagina

The upper uterine segment and ovaries appeared normal on MRI and a 1.0-cm left external iliac lymph node was appreciated. The patient underwent a metastatic workup including positron emission tomography (PET) imaging. PET imaging showed increased metabolic activity in cells on the cervical surface, corresponding to the cervical cancer as well as in the para-aortic and pelvic lymph nodes (Figure 3). 


\section{Cureus}

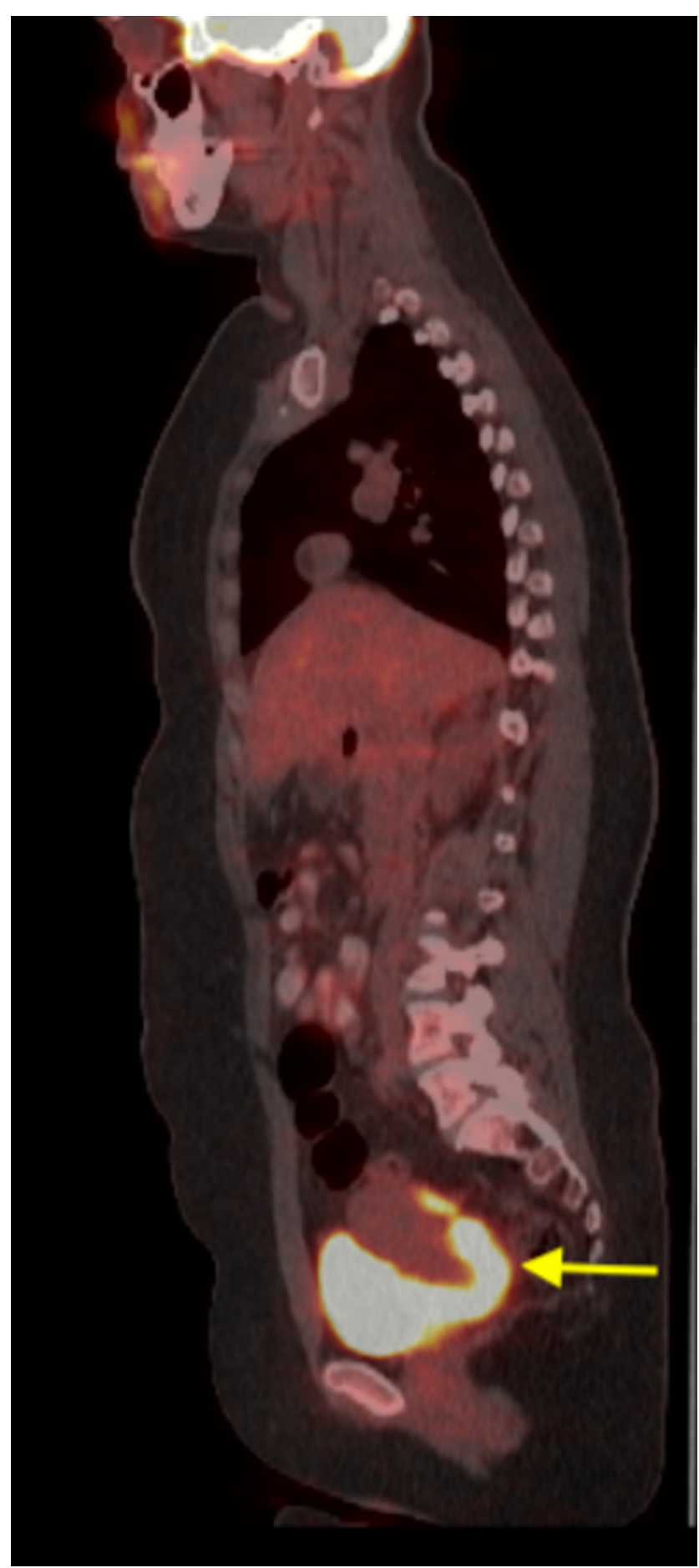

\section{FIGURE 3: Positron emission tomography scan showed increased}

metabolic activity in cells on the cervical surface

Also, there was an increased uptake in the bilateral ovaries, which raised the concern of ovarian metastasis versus a primary ovarian malignancy versus functional uptake. The patient underwent a bilateral salpingooophorectomy with omental/peritoneal biopsies and diaphragm smears. The subsequent pathology reports revealed no ovarian carcinoma. There was no evidence of malignancy in the omental/peritoneal biopsies and also the diaphragm smears. The patient was diagnosed with FIGO stage IB2 (T1b2N1M0) clear cell carcinoma of the cervix. She was treated with cisplatin at a dose of $30 \mathrm{mg} / \mathrm{m}^{2}$ and paclitaxel at a dose of 50 $\mathrm{mg} / \mathrm{m}^{2}$ for a total of eight cycles. She was also given concurrent external beam radiation therapy to the pelvic and para-aortic lymph nodes and also intracavitary brachytherapy. She received a total dose of 5,580 centigray (cGY) of radiation. She then underwent an MRI to assess treatment and she was found to have resolution of the cervical mass and also the para-aortic and pelvic lymph nodes (Figure 4). 


\section{Cureus}

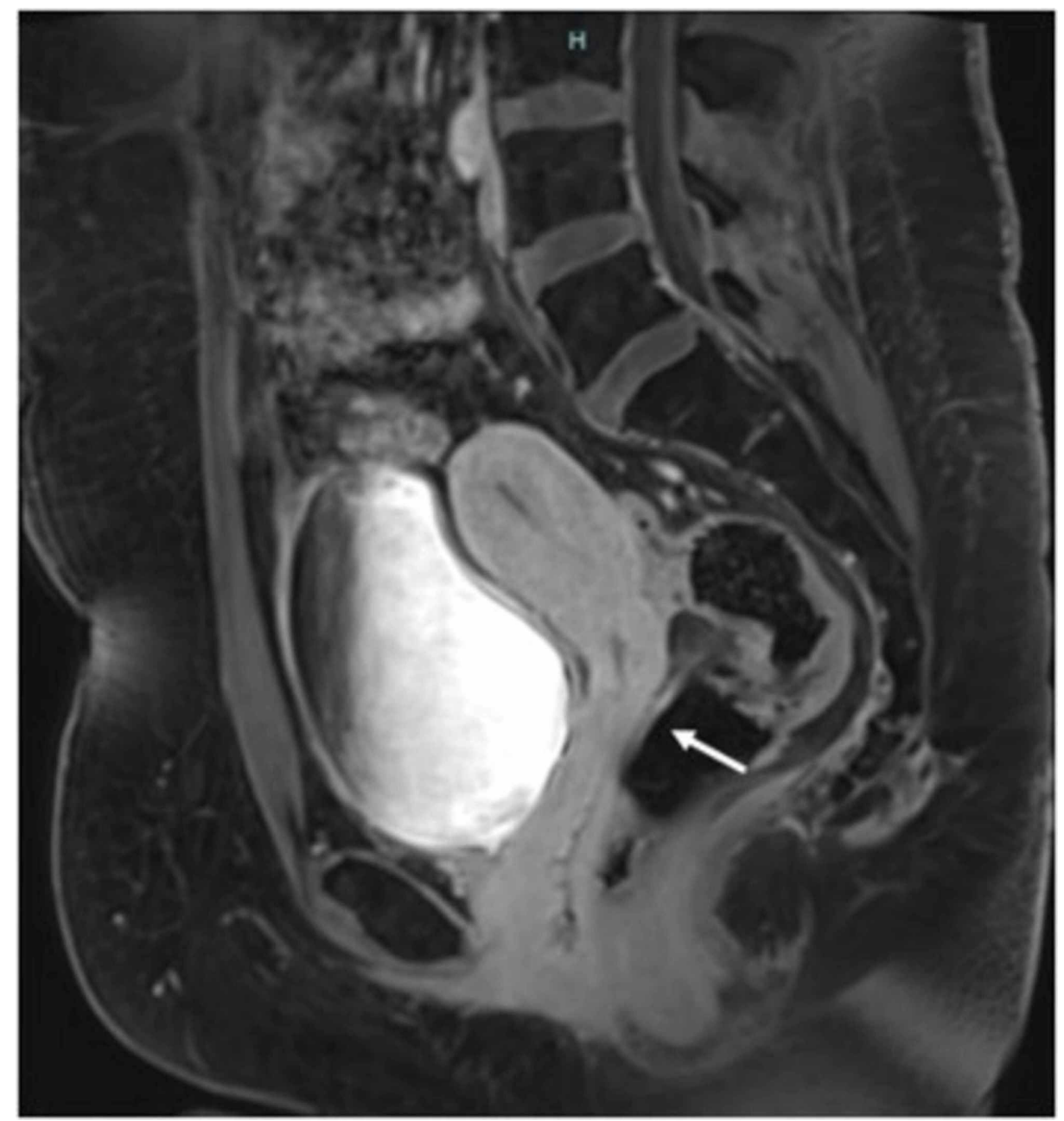

FIGURE 4: Pelvic MRI after treatment showing resolution of the cervical mass

The patient also underwent a PET scan after the treatment, and there was no evidence of local or distant metastatic disease (Figure 5). The patient continues to follow with oncology and has had a complete response to treatment and is currently nine months from the end of the combined therapy with no recurrence noted. 


\section{Cureus}

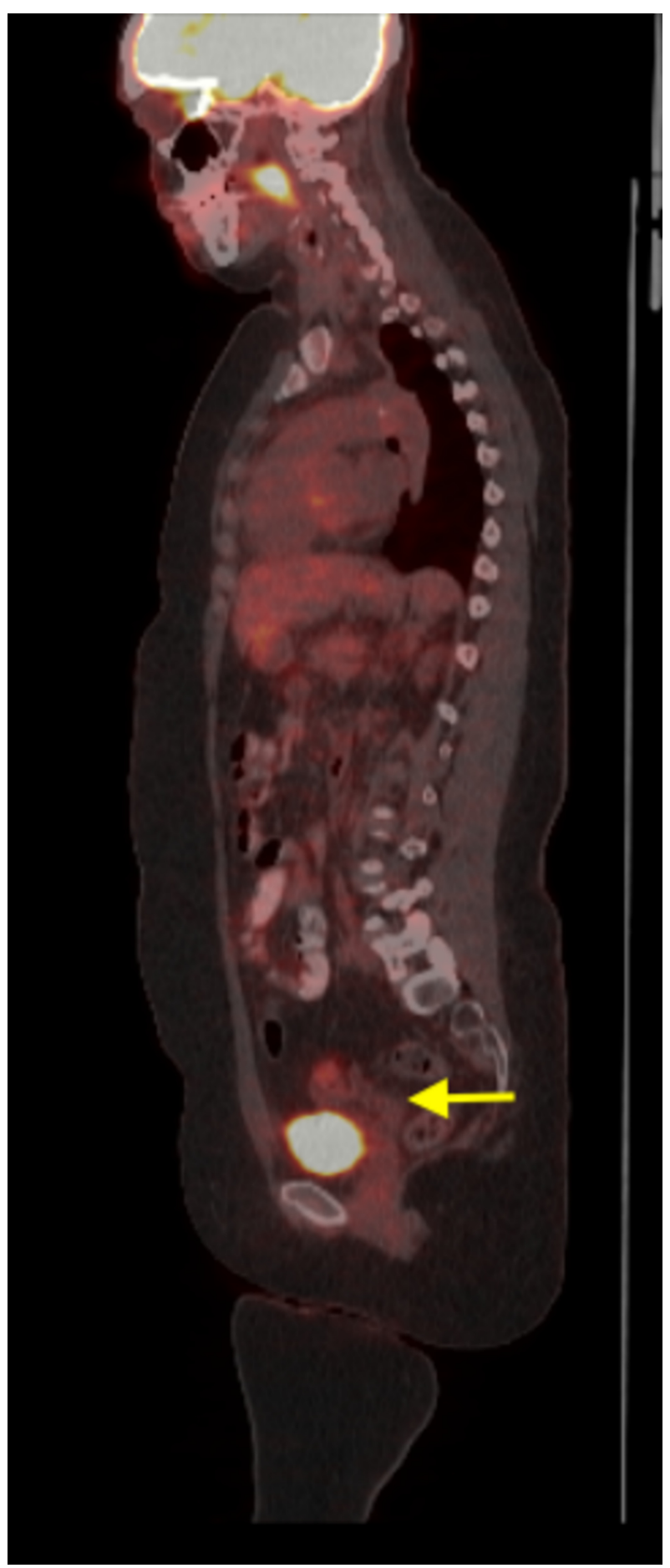

FIGURE 5: PET scan after treatment showing no evidence of local or distant metastatic disease

\section{Discussion}

Cervical cancer is the fourth most common cause of female cancers worldwide, both in terms of incidence and death toll [1]. There is significant geographical variability in the incidence and deaths caused by cervical cancer. Incidence is higher in areas with a lower Human Development Index, such as South East Asia and Sub-Saharan Africa [1].

With regard to the anatomy of the cervix, the transitional layer between the squamous and columnar epithelium, called the squamocolumnar junction, is believed to be at the greatest risk of malignant transformation. Squamous cell carcinomas of the cervix are more common than adenocarcinoma of the cervix, with the squamous cell variant accounting for $75 \%$ of invasive cervical cancers [1]. Clear cell 
One of the major risk factors associated with the development of CCAC was intrauterine DES exposure. DES was a synthetic estrogen that was used by pregnant women to prevent miscarriages, premature labor, and other pregnancy-related complications. In 1971, Herbst and colleagues demonstrated a clear association between the development of vaginal clear cell carcinoma and intrauterine DES exposure [2]. This led the FDA to ban the use of DES in pregnancy (in 1971). This further led to the establishment of the US Registry for Research on Hormonal Transplacental Carcinogenesis. Herbst, in 1999, reviewed 705 cases of clear cell adenocarcinoma which included both vaginal and cervical clear cell adenocarcinomas. Of these cases, $60 \%$ of cases had a clear exposure to intrauterine DES, $30 \%$ did not have DES exposure, and $10 \%$ had an unclear exposure history [7]. The etiology and risk factors for the development of non-DES related CCAC remain unclear. The risk factors associated with squamous cell carcinoma such as multiple sexual partners, HPV infection, increased age, and smoking are not associated with CCAC $[8,9]$. Pirog et al conducted a global study in which samples were collected from all over the world in order to determine the prevalence of HPV infection in different histological subtypes of cervical adenocarcinomas. Of the 682 cases of adenocarcinomas that were found to be eligible for the study, there were around 30 cases (4.4\%) of clear cell carcinoma. Of these 30 cases, six cases were found to be HPV positive [10]. Waggoner and colleagues in 1994 conducted a study to see if there was an association between HPV positivity and CCAC and vagina. However, there was no clear association that could be made [11]. It is unclear at this point whether HPV infection is a cofactor in the development of CCAC or just a coincidental finding. Further studies are required to find the association between CCAC and HPV infection. Cervical endometriosis has also been postulated as a risk factor for the development of CCAC since endometriosis is a risk factor for ovarian CCAC. There have been reports of CCAC arising in cervical endometriosis; however, no clear association is present [12]. p53 mutations and microsatellite repeats causing genomic instability have also been implicated in CCAC [13].

Hanselaar and colleagues analyzed the data registry in the Netherlands and noted that there was a twin peak incidence of CCAC at ages 26 and 71 years [14]. It is important to note that this included both DES-exposed and DES non-exposed patients. The early peak corresponded to patients who had been exposed to DES [14]. In a study done by Thomas and colleagues, which included $94 \%$ DES non-exposed patients, the mean peak incidence age was found to be 53 years [9]. This suggests that CCAC is more common in elderly postmenopausal women. Vaginal bleeding is the most common presentation of CCAC [9]. In pediatric patients, there can often be a delay in the diagnosis of CCAC. This could be because physicians feel hesitant performing per-vaginal exams in young girls and adolescents, and also because there is a tendency to diagnose young girls with vaginal bleeding with functional uterine bleeding [15]. It has been demonstrated that CCAC mostly shows an endophytic rather than an exophytic growth pattern, which can result in a normal pelvic exam [6]. This also explains the reason of a low rate of abnormal pap smears in CCAC, with some studies showing rates as low as $18 \%[6,9]$. CCAC shows different microscopic patterns, which include solid, tubulocystic, papillary, or a combination of features. In the solid pattern, the cells have cytoplasm which is clear and glycogen-rich with atypical nuclei. In the tubulocystic pattern, there are tubules and cystic spaces lined by clear cells. The papillary pattern is the least common type. The best outcome is associated with the tubulocystic pattern and the worst outcome with the solid pattern [8]. In a study by Reich and colleagues, it was identified that CCAC spread to the uterine corpus more commonly than other forms of cervical cancer [6]. This is important because spread to the uterine corpus portrays a negative prognosis. In the same study, it was noted that CCAC did not have an increased rate of spread to the vagina when compared to other types of cervical cancer. Common sites of lymphatic spread included the parametrial (40\%) and pelvic lymph nodes (47\%) [6]. Metastasis to the pelvic nodes increases the risk of involvement of para-aortic lymph nodes. In the study by Thomas and colleagues, it was noted that all patients with positive para-aortic lymph nodes had pelvic lymph node involvement [9]. Thus, it is suggested that para-aortic lymph node dissection be done in patients with pelvic lymph node involvement in CCAC [9]. Studies have suggested that ovarian metastasis is more common in adenocarcinoma of the cervix, but this is not applicable to CCAC. Studies have failed to show an increased risk of ovarian metastasis in CCAC [6]. Lung, liver, and bone are the extrapelvic sites of metastasis that have been identified [6].

The treatment of CCAC is not well defined and is largely based on the methods used to treat squamous cell carcinoma and non-clear cell adenocarcinoma. In the early stages, surgery is an option. There has been an increasing focus on fertility-preserving treatment. Vaginal radical trachelectomy and abdominal radical trachelectomy have emerged as viable options in early stages (IA-IB1) of cervical cancer without lymphatic spread, in women below 45 years of age and with a strong desire to preserve fertility [16]. In more advanced stages, surgery is not recommended, since it is unlikely that it will be curative. Also, advanced stages require adjuvant chemotherapy and radiotherapy, and if patients have had surgery, it is associated with a higher risk of complications [17]. In our patient as well, we did not proceed with surgery because of the advanced stage and was instead treated with chemotherapy and radiotherapy. The chemotherapy of choice is usually weekly cisplatin along with radiation. Combination chemotherapy with cisplatin and paclitaxel could also be an option, as in our patient, who had a complete response to the cisplatin and paclitaxel plus radiation. One study also suggested that there was an increased activation of the EGFR-PI3K-AKT-mTOR pathway in CCAC and that inhibitors of tyrosine kinase and AKT-mTOR may be novel therapeutic targets [18].

CCAC by itself does not indicate a poor prognosis. Reich and colleagues did not note any statistical difference in the five-year survival of CCAC when compared to squamous cell carcinoma of the cervix and 
non-CCAC [6]. The five-year survival rate for all stages of CCAC ranges from 40\% to $72.2 \%$ [19]. Poor prognostic factors include advanced-stage CCAC, with Thomas and colleagues reporting three-year survival rates as high as $91 \%$ in early-stage cervical cancer (stage I-stage IIA) compared to $22 \%$ in stage III or IV [9]. Hanselaar and colleagues noted that reported tumor size $>4 \mathrm{~cm}$ was an important negative prognostic indicator [14]. Nodal involvement has a negative impact on the overall survival (31\% vs 92\%) and progression-free survival (80\% vs 100\%) [10]. Nuclear atypia, increased mitotic activity, and a solid growth pattern all indicate a poor prognosis [9]. The median recurrence is around 12 months [10]. However, CCAC is known for late recurrences, with some studies reporting recurrences more than eight years after treatment [7]. This necessitates prolonged follow-up of patients with CCAC.

\section{Conclusions}

CCAC without DES exposure continues to be a rare disease. With its similarity of presentation to anovulatory bleeding in young women, and often presenting without a cervical mass, it requires an astute physician to clinch the diagnosis. With no clear guidelines for the management of CCAC, there needs to more randomized control trials for finding the appropriate treatment regimen. But the rarity of the condition might prove to be a challenge in conducting large-scale trials.

\section{Additional Information \\ Disclosures}

Human subjects: Consent was obtained by all participants in this study. Conflicts of interest: In compliance with the ICMJE uniform disclosure form, all authors declare the following: Payment/services info: All authors have declared that no financial support was received from any organization for the submitted work. Financial relationships: All authors have declared that they have no financial relationships at present or within the previous three years with any organizations that might have an interest in the submitted work. Other relationships: All authors have declared that there are no other relationships or activities that could appear to have influenced the submitted work.

\section{References}

1. Small W Jr, Bacon MA, Bajaj A, et al.: Cervical cancer: a global health crisis . Cancer. 2017, 123:2404-2412. 10.1002/cncr.30667

2. Herbst AL, Ulfelder H, Poskanzer DC: Adenocarcinoma of the vagina: association of maternal stilbestrol therapy with tumor appearance in young women. N Engl J Med. 1971, 284:878-881. 10.1056/NEJM197104222841604

3. Hasegawa K, Nagao S, Yasuda M, et al.: Gynecologic Cancer InterGroup (GCIG) consensus review for clear cell carcinoma of the uterine corpus and cervix. Int J Gynecol Cancer. 2014, 24:90-95. 10.1097/IGC.0000000000000297

4. Baykara M, Benekli M, Erdem O, et al.: Clear cell adenocarcinoma of the uterine cervix: a case report and review of the literature. J Pediatr Hematol Oncol. 2014, 36:131-133. 10.1097/MPH.0b013e318290cb1b

5. Jiang X, Jin Y, Li Y, et al.: Clear cell carcinoma of the uterine cervix: clinical characteristics and feasibility of fertility-preserving treatment. Onco Targets Ther. 2014, 7:111-116. 10.2147/OTT.S53204

6. Reich O, Tamussino K, Lahousen M, Pickel H, Haas J, Winter R: Clear cell carcinoma of the uterine cervix: pathology and prognosis in surgically treated stage IB-IIB disease in women not exposed in utero to diethylstilbestrol. Gynecol Oncol. 2000, 76:331-335. 10.1006/gyno.1999.5700

7. Herbst AL: Behavior of estrogen-associated female genital tract cancer and its relation to neoplasia following intrauterine exposure to diethylstilbestrol (DES). Gynecol Oncol. 2000, 76:147-156. 10.1006/gyno.1999.5471

8. Tantitamit T, Hamontri S, Rangsiratanakul L: Clear cell adenocarcinoma of the cervix in second generation young women who are without maternal exposure to diethylstilbestrol: a case report. Gynecol Oncol Rep. 2017, 20:34-36. 10.1016/j.gore.2017.02.008

9. Thomas MB, Wright JD, Leiser AL, et al.: Clear cell carcinoma of the cervix: a multi-institutional review in the post-DES era. Gynecol Oncol. 2008, 109:335-339. 10.1016/j.ygyno.2008.02.007

10. Pirog EC, Lloveras B, Molijn A, et al.: HPV prevalence and genotypes in different histological subtypes of cervical adenocarcinoma, a worldwide analysis of 760 cases. Mod Pathol. 2014, 27:1559. 10.1038/modpathol.2014.55

11. Waggoner SE, Anderson SM, Van Eyck S, Fuller J, Luce MC, Herbst AL: Human papillomavirus detection and p53 expression in clear-cell adenocarcinoma of the vagina and cervix. Obstet Gynecol. 1994, 84:404-408.

12. Hashiguchi M, Kai K, Nishiyama S, Nakao Y, Yokoyama M, Aishima S: Clear cell carcinoma of the uterine cervix presented as a submucosal tumor arising in a background of cervical endometriosis. Int J Gynecol Pathol. 2018, 37:88-92. 10.1097/PGP.0000000000000386

13. Boyd J, Takahashi H, Waggoner SE, et al.: Molecular genetic analysis of clear cell adenocarcinomas of the vagina and cervix associated and unassociated with diethylstilbestrol exposure in utero. Cancer. 1996, 77:507-513. 10.1002/(SICI)1097-0142(19960201)77:3<507::AID-CNCR12>3.0.CO;2-8

14. Hanselaar A, Loosbroek MV, Schuurbiers O, Helmerhorst T, Bulten J, Bernheim J: Clear cell adenocarcinoma of the vagina and cervix: an update of the central Netherlands registry showing twin age incidence peaks. Cancer. 1997, 79:2229-2236. 10.1002/(SICI)1097-0142(19970601)79:11<2229::AID-CNCR22>3.0.CO;2-X

15. McNall RY, Nowicki PD, Miller B, Billups CA, Liu T, Daw NC: Adenocarcinoma of the cervix and vagina in pediatric patients. Pediatr Blood Cancer. 2004, 43:289-294. 10.1002/pbc.20113

16. Gien LT, Covens A: Fertility-sparing options for early stage cervical cancer. Gynecol Oncol. 2010, 117:350357. 10.1016/i.ygyno.2010.01.039 


\section{Cureus}

17. Yamashita H, Okuma K, Kawana K, et al.: Comparison between conventional surgery plus postoperative adjuvant radiotherapy and concurrent chemoradiation for FIGO stage IIB cervical carcinoma: a retrospective study. Am J Clin Oncol. 2010, 33:583-586. 10.1097/COC.0b013e3181cae5b7

18. Ueno S, Sudo T, Oka N, et al.: Absence of human papillomavirus infection and activation of PI3K-AKT pathway in cervical clear cell carcinoma. Int J Gynecol Cancer. 2013, 23:1084-1091. 10.1097/IGC.0b013e3182981bdc

19. Yang L, Zheng A, Zhang X, Fang X, Sun W, Chen Y: Clear cell carcinoma of the uterine cervix: a clinical and pathological analysis of 47 patients without intrauterine diethylstilbestrol exposure. Int J Gynecol Cancer. 2017, 27:1009-1014. 10.1097/IGC.0000000000000992 